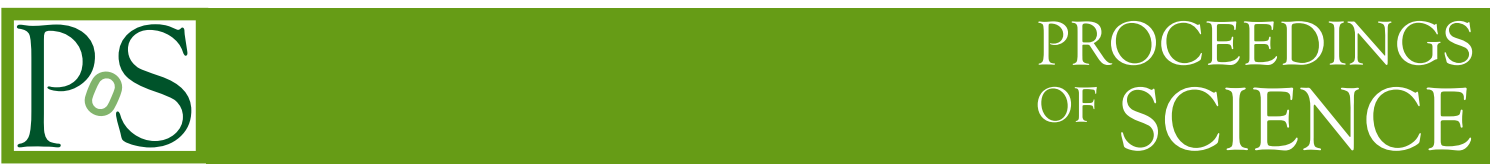

\title{
Dark Matter Theory
}

\section{Lars Bergström ${ }^{* \dagger}$}

The Oskar Klein Centre for Cosmoparticle Physics

Department of Physics, Stockholm University

AlbaNova, SE-106 91 Stockholm, Sweden

E-mail: lbedfysik.su.se

\begin{abstract}
The dark matter problems remains as one of the outstanding enigmas of particle physics and cosmology. Chances for solving the problem of the identity of dark matter depends on its nature, but for the set with supporting evidence from early universe theory, WIMPs (weakly interacting massive particles) there is great hope that the presently fast experimental progress may produce conclusive answers within a few years. A review of the current situation is made and it is argued that indications from various methods for detection are not yet strong enough to warrant claims of discovery. Prospects for the future are given, including some ideas of how to proceed if CERN's LHC would not discover a WIMP candidate during its present run.
\end{abstract}

The 2011 Europhysics Conference on High Energy Physics-HEP 2011,

July 21-27, 2011

Grenoble, Rhône-Alpes France

\footnotetext{
*Speaker.

${ }^{\dagger}$ Invited plenary talk.
} 


\section{Introduction}

We live in a universe where only $4-5 \%$ of the energy density is provided by ordinary matter. The remaining $95 \%$ is composed of two agents we still know very little about, gravitationally repulsive dark energy (72\%) and gravitationally attractive dark matter, DM, (23\%). Whereas additional knowledge about dark energy, whether is it a cosmological constant or a time-dependent expectation value of a scalar field, or something even more exotic, may take a decade or more to gather, dark matter may in this respect, in favourable cases, be more readily explorable. It can be argued that within a few years we will have tested most of the seemingly natural scenarios where the dark matter particles are thermodynamically produced in the early universe, in particular the WIMP scenario [1]. If we still have not found the solution by then, the problem may on the other hand well turn out to be at least as difficult as that of the dark energy.

One of the pioneers of dark matter research was Fritz Zwicky, who already in 1933 pointed out that galaxy clusters, in particular the Coma cluster, seem to have virial motion among the galaxies in the cluster which, based on the virial theorem, would mean that a much larger gravitating mass than the visible mass is present. We will later return to the potiential of galaxy clusters for pinning down the nature of the dark matter.

According to the last results from the WMAP satellite [2], the data are excellently described by the cosmological standard model, a flat $\Lambda$-dominated universe seeded by a nearly scale-invariant adiabatic Gaussian fluctuations, with the Hubble constant $h=0.72 \pm 0.05, \Omega_{B} h^{2}=0.024 \pm 0.001$, $\Omega_{M} h^{2}=0.14 \pm 0.02$. The difference between the matter density (normalized to the critical density) $\Omega_{M}$ and the baryon density $\Omega_{B}$, is given by cold dark matter with density $\Omega_{C D M}=0.23 \pm 0.04$. The problem for cosmology and particle physics is to explain this number, and to give candidates for the identity of the dark matter particles. The fact that dark matter is definitely needed on the largest scales (probed by WMAP), on galaxy cluster scales (as pointed out by Zwicky, and verified by gravitational lensing and the distribution of X-ray emitting gas) all the way down to the smallest dwarf galaxies, means that solutions based on changing the laws of gravity seems less natural. In particular, the direct empirical proof of the existence of dark matter given by the "Bullet Cluster" [3] is very difficult to circumvent, as the X-ray signal from the baryonic matter and the gravitational lensing signal from dark matter are clearly separated.

The particle physics connection is particularly striking in the WIMP scenario, namely that for typical gauge couplings and a mass at the weak interaction scale of a few hundred $\mathrm{GeV}$, the relic density computed using standard big bang thermodynamics (as tested, e.g., by the succesful calculaton of the abundances of light elements) turns out to be the cosmologically measured one. Although this is not a completely convincing argument for WIMP dark matter - it may perhaps be a coincidence - it nevertheless gives WIMP candidates a flavour of naturality. For non-WIMP candidates there is, on the other hand, usually a finetuning involved, or use of non-standard cosmology, to obtain the correct relic density. Even limiting oneself to WIMP models for dark matter, the literature is extensive, and among some recent developments, which cannot be discussed in this short review in any detail, can be mentioned:

- Dark stars. Since cosmological structure in WIMP models occurs hierarchically, starting from scales as small as $10^{-12}-10^{-6} m_{\text {sun }}$ [4], the idea has been put forward that the earliest 
dense, small structures created by dark matter may play a role in star formation and if the dark matter particles annihilate within the stars, unual stellar evolution may result [5].

- Inelastic dark matter. These are dark matter candidates which may excited to a state with slightly higher mass and therefore casue a higher than usual detection rate [6], and also relieve the tension between the different direct detection experiments (see later).

- Dynamical dark matter. As it is not obvious that there is only one type of particle making up the dark matter (neutrinos should, for example contribute up to a few percent), an extreme solution could be to have a very large number, with different spins, masses, etc. [7].

- Leptophilic dark matter. There was somewhat of an explosion of suggestions of this kind of models in 2009, when the dark matter interpretation of the anomalous positron ratio measured by PAMELA[8] and Fermi [9] was proposed to be explained by dark matter annihilation. Leptophilic means that these dark matter particles annihilate mainly to leptons, for example by proceeding through axion-like particles below the pion mass [10].

- Supersymmetric models beyond the MSSM [11]. These models may among other things give a higher Higgs mass than the limit of $130 \mathrm{GeV}$ given by minimal SUSY models.

- Asymmetric dark matter. This is a class of dark matter models which may also explain the baryon (or lepton) asymmetry of the universe [12]. This generally only works for masses around or below $10 \mathrm{GeV}$, and this mass range has been in focus recently due to a (possible) signal in direct detection experiments.

- Emergent dark matter. This is a version of asymmetric DM with larger possible parameter range, such as mass up to $100 \mathrm{GeV}$ [12].

WIMPs are arguably the leading candidates for Dark Matter, due to lack of fine-tuning to get correct relic density. In most models, the annihilation cross section which sets the relic density also implies observable rates in various DM detection experiments. A word of caution is in place here, however. There are many non-WIMP models that also have good particle physics motivation, and may be detectable, like: axions, gravitinos, superWIMPS, non-thermal dark matter, decaying dark matter, sterile Neutrinos, Q-balls... See, e.g., the recent 700-page extensive review of these as well as more standard WIMP models [14]. Noticeable progress the last few years has especially been made in axion searches [15]. However, this talk will deal mainly with a main template - WIMPs, and in particular SUSY WIMPs.

Supersymmetry, invented already in the 1970's, and obtained as a phenomenological manifestation of the most realistic string theories, has since the early 1980's, when the CDM paradigm first won universal acclaim, been the prime template for a WIMP [16, 17]. For a variety of reasons, the lightest neutralino in the $R$-parity conserving minimal supersymmetric standard model (MSSM),

$$
\widetilde{\chi}_{1}^{0}=a_{1} \widetilde{B}^{0}+a_{2} \widetilde{W}^{0}+a_{3} \widetilde{H}_{1}^{0}+a_{4} \widetilde{H}_{2}^{0},
$$

is the most natural choice explaining the dark matter. Even in the MSSM, however, there are in principle more than a hundred free parameters, meaning that for practical reasons the templates, 
for instance used at the LHC experiments, are drastically simplified versions (like CMSSM or the even more constrained mSUGRA), which do not, in contrast to the full MSSM, corrrespond very well to more recent thinking about supersymmetry breaking [18]. This has to be kept in mind when discussing the impressive LHC limits discussed extensively at this conference. Even in still simplified versions, like the 19 to 24-parameter "phenomenological MSSM" [19], the bounds on particle masses given, e.g., by fulfilling the WMAP relic density, are not very constraining at the moment [20]. Of course, the outlook for the MSSM would be much bleaker if a light Higgs (with mass below roughly $130 \mathrm{GeV}$ ) were not to be found by the end of the $7 \mathrm{TeV}$ run, in 2012 .

\section{Detection Methods for WIMPs}

There are basically three different, and complementary methods for detecting WIMPs. First, the dark matter particle may be directly produced at accelerators, in particular at the LHC, which today is the only high-energy accelerator running (although data from Fermilab's Tevatron collider will still be analyzed and may give surprises in the coming year or so). Of course, it is not clear that the particle will be kinematically allowed, and even if it is produced, one will not know that the lifetime is of the required cosmological order of magnitude. Anyway, detecting a candidate and determining its mass would be a great gain when combining with the other two search methods of dark matter, namely direct and indirect detection. In particular, direct detection experiments have seen an impressive gain of sensitivity during the last few years. The idea is to register rare events giving a combination of scintillation, ionization and nuclear recoil signals in chunks of matter shielded from cosmic rays in underground sites.

In indirect detection, one rather registers products of dark matter annihilation from regions in the surrounding universe with a high dark matter density like the galactic centre, dwarf spheroidal galaxies, or the interior of the Earth or the Sun. An interesting feature of indirect detection is that the expression for the local annihilation rate of a pair of DM particles $\chi$ (here assumed, like in supersymmetry, to be self-charge-conjugate, of relative velocity $v_{\text {rel }}$

$$
\Gamma_{a n n} \propto n_{\chi}^{2} \sigma_{a n n}\left(v_{r e l}\right) v_{r e l}
$$

is the dependence on the square of the number density. Also, the cross section may depend in non-trivial ways on the relative velocity. In particular, for low velocities the rate may be much higher than at high velocity, for models containing an attractive force between the annihilating particles. This is in particular true for models with so-called Sommerfeld enhancement [21], a resonant enhancement by in some cases orders of magnitude. This means that dwarf galaxies (dark matter subhalos) may be particulary intesting objects to study, as they are completely dark matter dominated with low rate of cosmic ray-induced gamma-rays, and their low mass means a relatively low velocity dispersion, meaning higher possible rates if Sommerfeld enhancement is active.

So far, indirect methods have not been as competitive as direct detection, but recently the Fermi collaboration has started to probe the interesting WIMP region by stacking data from several dwarf galaxies [22].

For non-WIMP dark matter, like sterile neutrinos (warm DM), the production rate in the early universe generally has to be tuned to give the observed relic density, but phenomenologically warm DM is possible, and according to some analyses even preferred in cosmological data [23]. However, 


\begin{tabular}{|l|l|}
\hline Experiment & Status of claim \\
\hline \hline DAMA/LIBRA annual modulation[25] & $\begin{array}{l}\text { Unexplained at the moment; not confirmed by } \\
\text { other experiments [26, 27] }\end{array}$ \\
\hline $\begin{array}{l}\text { CoGeNT excess events and annual modula- } \\
\text { tion [29] }\end{array}$ & Tension with other data [26, 27] \\
\hline EGRET excess of GeV photons [30,31] & $\begin{array}{l}\text { Due to instrument error (?) - not confirmed } \\
\text { by FERMI [32] }\end{array}$ \\
\hline $\begin{array}{l}\text { INTEGRAL 511 keV } \gamma \text {-line from galactic } \\
\text { centre region [33] }\end{array}$ & $\begin{array}{l}\text { Does not seem to have spherical symmetry - } \\
\text { shows an asymmetry which follows the disk } \\
\text { (?) [34] }\end{array}$ \\
\hline $\begin{array}{l}\text { PAMELA: Anomalous ratio of cosmic ray } \\
\text { positrons/electrons [8] }\end{array}$ & $\begin{array}{l}\text { May be due to DM [35], or pulsars [36] - en- } \\
\text { ergy signature not unique for DM }\end{array}$ \\
\hline FERMI positrons + electrons [9] & $\begin{array}{l}\text { May be due to DM [35], or pulsars [36] - en- } \\
\text { ergy signature not unique for DM }\end{array}$ \\
\hline $\begin{array}{l}\text { FERMI } \gamma \text {-ray excess towards galactic centre } \\
\text { [37] }\end{array}$ & $\begin{array}{l}\text { Unexplained at the moment - astrophysical } \\
\text { explanations possible [38, 39], no statement } \\
\text { from the FERMI collaboration }\end{array}$ \\
\hline WMAP radio "haze" [40] & $\begin{array}{l}\text { Has a correspondence in "FERMI bubbles" } \\
\text { [41] - probably caused by outflow from the } \\
\text { galactic center }\end{array}$ \\
\hline
\end{tabular}

Table 1: Some of the recent experimental claims for possible dark matter detection, and a comment on the present status.

the significance is weak and may be influenced by statistical bias [24]. Ordinary, active neutrinos have too small mass to contribute significantly to the dark matter density, although in the extreme case may contribute a couple of percent to the critical density today.

There have recently been a number of claimed possible detections of dark matter, see Table 1 . Of the items in Table 1, it seems that only the positron excess at high energy ( $20 \mathrm{GeV}-1 \mathrm{TeV}$ ) and the $\gamma$-ray excess towards the galactic center, inferred by an anlysis of FERMI public data [37], may have a chance to be due to dark matter annihilation. However, they may both perhaps more naturally be explained by ordinary astrophysical processes. In addition, the DM explanation of the PAMELA and FERMI data needs a leptophilic particle of TeV-scale mass and a very much boosted cross section. Although this may perhaps be obtained, stretching all uncertainties involved [42], and employing Sommerfeld enhancement [43], the remaining window seems quite tight.

The DAMA/LIBRA annual modulation is a statistically very strong signal (significance of the order of $8 \sigma$ ), however the lack of supporting data from other experiments is disturbing. The annual modulation hinted at by CoGeNT [29] is statistically much weaker, and the purported excess unmodulated signal may in fact be incompatible with the level of modulated reported. Also, it seems that the DAMA/LIBRA and GoGeNT signals, if interpreted as being due to dark matter, may 
be in tension with each other, even if one uses freedom in isospin violation, inelastic scattering, and non-standard halo properties [44]. At the moment this is one of the unsolved, frequently debated issues in the dark matter community.

The recent improvement of the upper limits on the WIMP-nucleon scattering cross section reported by CDMS II [26] and, in particular, XENON100 [27] are truly impressive. Not only does it cast some doubt on other reported experimental results, the sensitivity is also good enough to start probing the parameter space of supersymmetric models [28]. The new calibration of the sensitivity to low-energy recoils of Xenon adds to the credibility of the new limits. The very good news is also that the installation of the next stage, a 1 ton liquid Xenon detector, has already started in the Gran Sasso experimental halls in Italy.

A somewhat different, and complementary, method is indirect detecton of neutrinos. As the most of the elements in the interior of the Earth have spin zero, capture of WIMPS in the Earth takes places through the same type of spin-independent scattering that is used bin the CDMS and XENON100 detectors. Therefore, the neutrino limits from the interior of the Earth are not competitive at the moment. However, the Sun consists mostly of hydrogen which means that spindependent scattering on protons will be important for the capture rate, and consequently for the annnihilation rate, from the Sun. The spin-dependent limits thus obtained are in many cases superior to present-day direct detection limits on spin-dependent scattering [46], especially with the low-threshold inset Deep-Core and the full 80-string outer IceCube detector now in place.

Antimatter does not seem to be present in large quantities in the universe, as can be inferred from the absence of $\gamma$-ray radiation that would have been created in large amounts if astrophysical anti-objects would annihilate on their matter counterparts (this would also cause deviations from the pure black-body form of the cosmic microwave background). In fact, both the analysis of primordial nucleosynthesis and the $\mathrm{CMB}$, give non-zero number around $10^{-10}$ for the baryonantibaryon asymmetry, which means that matter dominated over antimatter already in the very early universe. On the other hand, dark matter annihilation occurs from a matter-antimatter symmetric initial state and thus equal amounts of matter and antimatter would be created, leading to an interesting possible primary source of positrons and antiprotons (i.e. stable anti-particles) in the cosmic rays of dark matter halos, including the one where the Milky Way resides. (There is always a small amount of antimatter produced as secondary particles in collisions with galactic gas and dust by ordinary cosmic rays, of course.) As discussed in the EPS-HEP conference two years ago [47] this was an extremely hot topic then, as the PAMELA and FERMI collaborations had just discovered an anomalously high ratio of positrons over electrons up to $100 \mathrm{GeV}$ [8], and sum of positrons and electrons up to $1 \mathrm{TeV}$ [9], respectively. During the last two years, this anomaly, although possible to explain by dark matter annihilation, needs such large boost factors (e.g., from Sommerfeld enhancement), and the somewhat contrived, leptophilic models, whereas astrophysical explanations are possible with quite standard assumptions. One cannot say that the dark matter explanation is yet ruled out, but it sees tension from other measurements, especially from gamma-rays.

Returning to more standard WIMP models, there have recently been improvements in the computations of the annihilation rate at low velocity as is the case in galaxies, where $v / c \sim 10^{-3}$. An amusing effect is caused due to the suppression of the ${ }^{3} S_{1}$ for an initial initial state of two Majorana spinors (such as neutralinos) at zero velocity, due to the requirement of Femi statistics for the two idenitcal fermions if they are in the same spin state. This means that annihilation only 
occurs from the pseudoscalar ${ }^{1} S_{0}$ state, causing for instance the annihilation amplitude into a light fermion-anntifermion pair, like $e^{+} e^{-}$, to be suppressed by an explicit helicity factor of the fermion mass. Direct annihilation into $e^{+} e^{-}$was thus thought to be very subdominant. However, it was realised [48] (building on an old idea [49]), that a spin-flip by one of the Majorana fermions caused by emitting a photon could first of all relieve the helicity suppression of the process to a mere $\alpha / \pi$ ordinariy radiative factor. And, in addition, the spectral shape of the emitted photon is very favourable for detection, causing a shoulder which peaks close to the dark matter particle mass. In particular, for heavy (TeV-scale) WIMPs this could be quite important, and using the radiative peak would help extracting the signal over background [50]. Recently, these radiative processes have been generalized also to emission of other gauge bosons, and have been shown to be quite important generally [51].

One dificulty when estimating gamma-ray rates from dark matter annihilation is the poorly known distribution of dark matter on galactic and subgalactic scales. $N$-body simulations indicate that the halo should be very abundant with dark matter clumps, but since the rate is a line-of sight integral along a given directon which is sensitive to the square of the local density along the way,

$$
\frac{\Phi_{\gamma}(\psi)}{\mathrm{cm}^{-2} \mathrm{~s}^{-1} \mathrm{sr}^{-1}} \simeq 0.94 \cdot 10^{-13}\left(\frac{N_{\gamma}<\sigma v>}{10^{-29} \mathrm{~cm}^{3} \mathrm{~s}^{-1}}\right)\left(\frac{100 \mathrm{GeV}}{M_{\chi}}\right)^{2} J(\psi)
$$

with the astrophysical part residing in the dimensionless function

$$
J(\psi)=\frac{1}{8.5 \mathrm{kpc}} \cdot\left(\frac{1}{0.3 \mathrm{GeV} / \mathrm{cm}^{3}}\right)^{2} \int_{\text {l.o.s. }} \rho^{2}(l) d l(\psi),
$$

even enhancements of the density on very small scales may be important. An example is the region near the galactic centre, where gravity is dominated by the black hole and a stellar cusp, with unknown effects on the annihilation rate into $\gamma$-rays. Unfortunately, the contribution from dark matter to the rotation curve is much too small to enable to determine if the halo density is cuspy, as favoured by the results of $\mathrm{N}$-body dark matter-only simulations, or if it has a milder dependence or even a core [52]. As a simple template of the DM distribution, an NFW profile [53] having an $1 / r$ cusp near the center is often used, but it is as said unknown whether the real distribution is more cuspy or less, and predictions vary by several orders of magnitude due to this uncertainty. Even if the center of the galaxy plausibly is the most interesting place to search for gamma rays from DM annihilation, fore- and backgrounds from astrophysical processes may be large, and thus it may be advantageous to search in directions close to, but not exactly at, the galactic center [54].

Interesting objects are the dwarf galaxies mentioned above, there FERMI limits are now getting close to the predicted WIMP cross section [22]. The abundance of DM clumps may in fact be much higher, as star formation probably only occurs above some threshold mass, and the pressure from a few supernovae may be enough to empty a dwarf galaxy from baryons. Simulations indicate that DM clumps will be destroyed by tidal forces near the center of galaxies but can be very aboundant in the outer regions [55]. It also seems that when going to larger scale objects like galaxy clusters, the number of undestroyed DM clumps may be even larger, making these clusters - in a perhaps unexpected agreement with the discovery of Zwicky - quite promising targets for indirect searches [56]. 
In the first runs at LHC, no signs of a Higgs particle, nor supersymmetry or any other of the prime candidates for dark matter, have been discovered. On the other hand, the mass region 115 $130 \mathrm{GeV}$, interesting for the lightest Higgs boson in the simplest versions of supersymmetry, has yet to be investigated. One possible scenario might be that such a Higgs particle is indeed found, but the particles carrying non-trivial $R$-parity all have masses beyond reach with the LHC. This is not impossible, depending on the amount of fine-tuning one is willing to tolerate. In fact, if one puts no prior constraints on the supersymmetric parameter space other than one should have the WMAP-measured relic density, and fulfil all other experimental constraints ( $c f$. [20]), a mass for the lightest supersymmetric neutralino in the $\mathrm{TeV}$ region is generic. For such heavy dark matter neutralinos, the rate for direct detection will also be small, and it would seem impossible to test such a scenario. However, for this particular case indirect detection through gamma rays turns out to have an interesting advantage, as the new imaging air Chererenkov arrays like CTA [57] will have their peak sensitivity in the energy range between a few hundred GeV to a few $\mathrm{TeV}$. Depending on the particular model realized in nature, Sommerfeld enhancement of indirect detection may also be operative. However, these large arrays will be served by a large astrophysical community which will be very much interested in transient or periodic events, meaning that a "boring" search for a stationary dark matter spectral signature during hundreds or even thousands of hours seem out of the question. One may therefore consider a dedicated particle physics experiment, the "Dark Matter Array", DMA [58] only used for dark matter search. This would have great, and complementary, potential to the large direct detection experiments that are presently being planned. In fact, there are ideas [59] on how to decrease the lower threshold for detection, something that could increase the sensitivity for DM detection considerably. If a working prototype of this type could be built, this idea may materialize in the next decade as a new way to search for phenomena beyond the Standard Model - with an expensive dedicated detector, still far below the cost of a new highenergy accelerator.

\section{Acknowledgements}

I wish to thank my colleagues, especially Felix Aharonian, Torsten Bringmann, Jan Conrad, and Joakim Edsjö, for many useful discussions. This research was carried out under Swedish Science Research Council contract no. 621-2009-3915.

\section{References}

[1] G. Bertone, Nature 468 (2010) 389-393. [arXiv:1011.3532 [astro-ph.CO]].

[2] E. Komatsu et al. [ WMAP Collaboration ], Astrophys. J. Suppl. 192 (2011) 18. [arXiv:1001.4538 [astro-ph.CO]].

[3] D. Clowe, M. Bradac, A. H. Gonzalez, M. Markevitch, S. W. Randall, C. Jones, D. Zaritsky, Astrophys. J. 648 (2006) L109-L113. [astro-ph/0608407].

[4] T. Bringmann, New J. Phys. 11 (2009) 105027. [arXiv:0903.0189 [astro-ph.CO]].

[5] D. Spolyar, K. Freese, P. Gondolo, Phys. Rev. Lett. 100 (2008) 051101. [arXiv:0705.0521 [astro-ph]]; F. Iocco, Astrophys. J. 677, L1-L4 (2008). [arXiv:0802.0941 [astro-ph]] P. Scott, M. Fairbairn, J. Edsjo, Mon. Not. Roy. Astron. Soc. 394 (2009) 82. [arXiv:0809.1871 [astro-ph]]; S. Sivertsson, P. Gondolo, Astrophys. J. 729 (2011) 51. [arXiv:1006.0025 [astro-ph.CO]]. 
[6] See, e.g., D. Tucker-Smith, N. Weiner, Phys. Rev. D64 (2001) 043502. [hep-ph/0101138]; J. March-Russell, C. McCabe, M. McCullough, JHEP 0905 (2009) 071. [arXiv:0812.1931 [astro-ph]]; Y. Cui, D. E. Morrissey, D. Poland, L. Randall, JHEP 0905 (2009) 076. [arXiv:0901.0557 [hep-ph]]; D. P. Finkbeiner, T. Lin, N. Weiner, Phys. Rev. D80 (2009) 115008. [arXiv:0906.0002 [astro-ph.CO]]; D. S. M. Alves, M. Lisanti, J. G. Wacker, Phys. Rev. D82 (2010) 031901. [arXiv:1005.5421 [hep-ph]]; M. T. Frandsen, F. Kahlhoefer, J. March-Russell, C. McCabe, M. McCullough, K. Schmidt-Hoberg, Phys. Rev. D84 (2011) 041301. [arXiv:1105.3734 [hep-ph]].

[7] See, e.g., K. R. Dienes, B. Thomas, [arXiv:1106.4546 [hep-ph]].

[8] O. Adriani et al. [ PAMELA Collaboration ], Nature 458 (2009) 607-609. [arXiv:0810.4995 [astro-ph]].

[9] A. A. Abdo et al. [ The Fermi LAT Collaboration ], Phys. Rev. Lett. 102 (2009) 181101. [arXiv:0905.0025 [astro-ph.HE]].

[10] Y. Nomura, J. Thaler, Phys. Rev. D79 (2009) 075008. [arXiv:0810.5397 [hep-ph]]; P. J. Fox, E. Poppitz, Phys. Rev. D79 (2009) 083528. [arXiv:0811.0399 [hep-ph]]; B. Kyae, JCAP 0907 (2009) 028. [arXiv:0902.0071 [hep-ph]]; D. Spolyar, M. R. Buckley, K. Freese, D. Hooper, H. Murayama, [arXiv:0905.4764 [astro-ph.CO]]; A. Ibarra, A. Ringwald, D. Tran, C. Weniger, JCAP 0908 (2009) 017. [arXiv:0903.3625 [hep-ph]]; T. Cohen, K. M. Zurek, Phys. Rev. Lett. 104 (2010) 101301. [arXiv:0909.2035 [hep-ph]]; N. Haba, Y. Kajiyama, S. Matsumoto, H. Okada, K. Yoshioka, Phys. Lett. B695 (2011) 476-481. [arXiv:1008.4777 [hep-ph]].

[11] See, e.g., A. Menon, D. E. Morrissey, C. E. M. Wagner, Phys. Rev. D70 (2004) 035005. [hep-ph/0404184]; M. Dine, N. Seiberg, S. Thomas, Phys. Rev. D76 (2007) 095004. [arXiv:0707.0005 [hep-ph]]; N. Bernal, A. Goudelis, JCAP 1003 (2010) 007. [arXiv:0912.3905 [hep-ph]]; M. Berg, J. Edsjo, P. Gondolo, E. Lundström, S. Sjors, JCAP 0908 (2009) 035. [arXiv:0906.0583 [hep-ph]].

[12] See, e.g., D. B. Kaplan, Phys. Rev. Lett. 68 (1992) 741-743; D. E. Kaplan, M. A. Luty, K. M. Zurek, Phys. Rev. D79 (2009) 115016. [arXiv:0901.4117 [hep-ph]]; A. L. Fitzpatrick, D. Hooper,

K. M. Zurek, Phys. Rev. D81, 115005 (2010). [arXiv:1003.0014 [hep-ph]; M. T. Frandsen, S. Sarkar, Phys. Rev. Lett. 105 (2010) 011301. [arXiv:1003.4505 [hep-ph]]; M. L. Graesser, I. M. Shoemaker, L. Vecchi, [arXiv:1103.2771 [hep-ph]]; S. Profumo, L. Ubaldi, JCAP 1108 (2011) 020. [arXiv:1106.4568 [hep-ph]].

[13] Y. Cui, L. Randall, B. Shuve, [arXiv:1106.4834 [hep-ph]].

[14] G. Bertone, (ed.), "Particle dark matter: Observations, models and searches," Cambridge University Press (2010) 738 p.

[15] S. J. Asztalos et al. [ The ADMX Collaboration ], Phys. Rev. Lett. 104 (2010) 041301. [arXiv:0910.5914 [astro-ph.CO]].

[16] H. Goldberg, Phys. Rev. Lett. 50 (1983) 1419.

[17] J. R. Ellis, J. S. Hagelin, D. V. Nanopoulos, K. A. Olive, M. Srednicki, Nucl. Phys. B238 (1984) 453-476.

[18] P. Meade, N. Seiberg, D. Shih, Prog. Theor. Phys. Suppl. 177, 143-158 (2009). [arXiv:0801.3278 [hep-ph]].

[19] C. F. Berger, J. S. Gainer, J. L. Hewett, T. G. Rizzo, JHEP 0902, 023 (2009). [arXiv:0812.0980 [hep-ph]]; J. A. Conley, J. S. Gainer, J. L. Hewett, M. P. Le, T. G. Rizzo, [arXiv:1103.1697 [hep-ph]]; 
[20] S. S. AbdusSalam, B. C. Allanach, H. K. Dreiner, J. Ellis, U. Ellwanger, J. Gunion, S. Heinemeyer, M. Kraemer et al., [arXiv:1109.3859 [hep-ph]].

[21] J. Hisano, S. .Matsumoto, M. M. Nojiri, O. Saito, Phys. Rev. D71 (2005) 063528. [hep-ph/0412403]; N. Arkani-Hamed, D. P. Finkbeiner, T. R. Slatyer, N. Weiner, Phys. Rev. D79 (2009) 015014. [arXiv:0810.0713 [hep-ph]].

[22] M. L. Garde, [arXiv:1102.5701 [astro-ph.HE]]; M. Ackermann et al. [The Fermi LAT Collaboration], [arXiv:1109.0521 [astro-ph.HE]].

[23] J. Hamann, S. Hannestad, G. G. Raffelt, I. Tamborra, Y. Y. Y. Wong, Phys. Rev. Lett. 105 (2010) 181301. [arXiv:1006.5276 [hep-ph]].

[24] A. X. Gonzalez-Morales, R. Poltis, B. D. Sherwin, L. Verde, [arXiv:1106.5052 [astro-ph.CO]].

[25] R. Bernabei et al. [ DAMA Collaboration ], Eur. Phys. J. C56 (2008) 333-355. [arXiv:0804.2741 [astro-ph]].

[26] Z. Ahmed et al. [ The CDMS-II Collaboration ], Science 327 (2010) 1619-1621. [arXiv:0912.3592 [astro-ph.CO]].

[27] E. Aprile et al. [ XENON100 Collaboration ], Phys. Rev. Lett. 105 (2010) 131302. [arXiv:1005.0380 [astro-ph.CO]].

[28] P. Gondolo, J. Edsjö, P. Ullio, L. Bergström, M. Schelke, E. A. Baltz, JCAP 0407 (2004) 008. [astro-ph/0406204].

[29] C. E. Aalseth et al. [ CoGeNT Collaboration ], Phys. Rev. Lett. 106 (2011) 131301. [arXiv:1002.4703 [astro-ph.CO]]; C. E. Aalseth, P. S. Barbeau, J. Colaresi, J. I. Collar, J. Diaz Leon, J. E. Fast, N. Fields, T. W. Hossbach et al., Phys. Rev. Lett. 107 (2011) 141301. [arXiv:1106.0650 [astro-ph.CO]].

[30] S. D. Hunter, D. L. Bertsch, J. R. Catelli, T. M. Digel, S. W. Dingus, J. A. Esposito, C. E. Fichtel, C. E. Hartman et al., Astrophys. J. 481 (1997) 205-240.

[31] W. de Boer, C. Sander, V. Zhukov, A. V. Gladyshev, D. I. Kazakov, Astron. Astrophys. 444 (2005) 51. [astro-ph/0508617].

[32] A. A. Abdo et al. [ The Fermi-LAT Collaboration ], Phys. Rev. Lett. 104 (2010) 101101. [arXiv:1002.3603 [astro-ph.HE]].

[33] J. Knodlseder, P. Jean, V. Lonjou, G. Weidenspointner, N. Guessoum, W. Gillard, G. Skinner, P. von Ballmoos et al., Astron. Astrophys. 441 (2005) 513-532. [astro-ph/0506026].

[34] G. Weidenspointner, G. Skinner, P. Jean, J. Knodlseder, P. von Ballmoos, G. Bignami, R. Diehl, A. W. Strong et al., Nature 451 (2008) 159-162.

[35] See, e.g., P. Meade, M. Papucci, A. Strumia, T. Volansky, Nucl. Phys. B831 (2010) 178-203. [arXiv:0905.0480 [hep-ph]]; L. Bergström, J. Edsjö, G. Zaharijas, Phys. Rev. Lett. 103 (2009) 031103. [arXiv:0905.0333 [astro-ph.HE]].

[36] F. A. Aharonian, A. M. Atoyan, H. J. Völk, Astron. Astrophys. 294 (1995) L41-L44; D. Grasso et al. [ FERMI-LAT Collaboration ], Astropart. Phys. 32 (2009) 140-151. [arXiv:0905.0636 [astro-ph.HE]].

[37] D. Hooper, L. Goodenough, Phys. Lett. B697 (2011) 412-428. [arXiv:1010.2752 [hep-ph]].

[38] M. Chernyakova, D. Malyshev, F. A. Aharonian, R. M. Crocker, D. I. Jones, Astrophys. J. 726 (2011) 60. [arXiv:1009.2630 [astro-ph.HE]]. 
[39] A. Boyarsky, D. Malyshev, O. Ruchayskiy, [arXiv:1012.5839 [hep-ph]].

[40] D. Hooper, D. P. Finkbeiner, G. Dobler, Phys. Rev. D76 (2007) 083012. [arXiv:0705.3655 [astro-ph]].

[41] M. Su, T. R. Slatyer, D. P. Finkbeiner, Astrophys. J. 724 (2010) 1044-1082. [arXiv:1005.5480 [astro-ph.HE]].

[42] G. Bertone, M. Cirelli, A. Strumia, M. Taoso, JCAP 0903 (2009) 009. [arXiv:0811.3744 [astro-ph]].

[43] D. P. Finkbeiner, L. Goodenough, T. R. Slatyer, M. Vogelsberger, N. Weiner, JCAP 1105 (2011) 002. [arXiv:1011.3082 [hep-ph]].

[44] T. Schwetz, J. Zupan, JCAP 1108 (2011) 008. [arXiv:1106.6241 [hep-ph]];

[45] P. J. Fox, J. Kopp, M. Lisanti, N. Weiner, [arXiv:1107.0717 [hep-ph]]; M. Farina, D. Pappadopulo, A. Strumia, T. Volansky, [arXiv:1107.0715 [hep-ph]].

[46] F. Halzen, D. Hooper, New J. Phys. 11 (2009) 105019. [arXiv:0910.4513 [astro-ph.HE]].

[47] A. Strumia, PoS EPS-HEP2009 (2009) 012.

[48] T. Bringmann, L. Bergström, J. Edsjö, JHEP 0801 (2008) 049. [arXiv:0710.3169 [hep-ph]]; L. Bergström, T. Bringmann, J. Edsjö, Phys. Rev. D78 (2008) 103520. [arXiv:0808.3725 [astro-ph]].

[49] L. Bergström, Phys. Lett. B225 (1989) 372.

[50] T. Bringmann, F. Calore, G. Vertongen, C. Weniger, [arXiv:1106.1874 [hep-ph]].

[51] N. F. Bell, J. B. Dent, A. J. Galea, T. D. Jacques, L. M. Krauss, T. J. Weiler, [arXiv:1104.3823 [hep-ph]]; P. Ciafaloni, M. Cirelli, D. Comelli, A. De Simone, A. Riotto, A. Urbano, JCAP 1110 (2011) 034. [arXiv:1107.4453 [hep-ph]].

[52] Y. Sofue, M. Honma, T. Omodaka, PASJ 61 (2008) 227. [arXiv:0811.0859 [astro-ph]].

[53] J.F. Navarro, C.S. Frenk and S.D.M. White, Astrophys. J. 462 (1996) 563.

[54] E. A. Baltz, B. Berenji, G. Bertone, L. Bergström, E. Bloom, T. Bringmann, J. Chiang, J. Cohen-Tanugi et al., JCAP 0807 (2008) 013. [arXiv:0806.2911 [astro-ph]].

[55] V. Springel, S. D. M. White, C. S. Frenk, J. F. Navarro, A. Jenkins, M. Vogelsberger, J. Wang, A. Ludlow et al., Nature 456N7218 (2008) 73-80.

[56] T. E. Jeltema, J. Kehayias, S. Profumo, Phys. Rev. D80 (2009) 023005. [arXiv:0812.0597 [astro-ph]]; M. A. Sanchez-Conde, M. Cannoni, F. Zandanel, M. E. Gomez, F. Prada, [arXiv:1104.3530 [astro-ph.HE]]; A. Pinzke, C. Pfrommer, L. Bergström, [arXiv:1105.3240 [astro-ph.HE]]; L. Gao, C. S. Frenk, A. Jenkins, V. Springel, S. D. M. White, [arXiv:1107.1916 [astro-ph.CO]].

[57] Cherenkov Telescope Array Consortium, [arXiv:1008.3703 [astro-ph.IM]].

[58] L. Bergström, T. Bringmann, J. Edsjö, Phys. Rev. D83 (2011) 045024. [arXiv:1011.4514 [hep-ph]].

[59] F. A. Aharonian, A. K. Konopelko, H. J. Volk, H. Quintana, Astropart. Phys. 15 (2001) 335-356. [astro-ph/0006163]. 